\title{
Sincronização da Onda Folicular com Buserelina Prévia à Indução da Luteólise com Cloprostenol em Bovinos
}

\author{
Deiler Sampaio Costa ${ }^{1}$, Ciro Alexandre Alves Torres ${ }^{2}$, Carlos Antônio de Carvalho Fernandes ${ }^{3}$, \\ Luiz Arthur C. Junqueira ${ }^{4}$, Cláudia D‘avila de Almeidaa ${ }^{4}$, Sandro Campos Alvim ${ }^{4}$
}

\begin{abstract}
RESUMO - Este experimento foi realizado para verificar o efeito da aplicação de diferentes doses de um análogo de GnRH (buserelina) sobre a emergência de nova onda de crescimento folicular e o número de folículos recrutados dessa onda, a formação de corpo lúteo acessório, o diâmetro do folículo dominante e do corpo lúteo espontâneo no dia da aplicação do luteolítico e subseqüente taxa de sincronização e o intervalo da aplicação de $\mathrm{PGF}_{2} \alpha$ à manifestação do estro. Vinte fêmeas bovinas em diestro, mestiças Holandês x Zebu e Simental x Zebu foram, distribuídas em quatro tratamentos contendo cinco animais cada. No tratamento 1, os animais receberam 4 mg IM de buserelina no dia z 0 (dia em que se iniciou o tratamento) e $150 \mathrm{mg}$ de $\mathrm{D}(+$ ) cloprostenol no dia 6 . Nos tratamentos 2,3 e 4 , receberam, respectivamente, 6,8 e $10 \mathrm{mg}$ de buserelina, pela mesma via de aplicação, e $150 \mathrm{mg}$ de $\mathrm{D}(+)$ cloprostenol no dia 6 . A atividade ovariana foi monitorada diariamente, com auxílio do aparelho de ultra-sonografia, do dia 0 ao estro, ou até o dia 11 pós-tratamento, naqueles animais que não responderam à prostaglandina. A aplicação de 8 ou $10 \mathrm{mg}$ de buserelina em fase indeterminada do diestro, nas fêmeas bovinas mestiças, promoveu emergência de nova onda de crescimento folicular mais rápida que aplicações de 4 ou 6 mg do mesmo hormônio; enquanto os outros parâmetros avaliados não diferiram entre os animais dos tratamentos.
\end{abstract}

Palavras-chave: dinâmica folicular, buserelina, cloprostenol, sincronização do estro, bovinos

\section{Follicular Wave Synchronization with Buserelin before the Luteolisis Induction with Cloprostenol in Cattle}

\begin{abstract}
This experiment was carried out to verify the effect of the administration of different doses of GnRH (buserelin) analogue on the emergence of a new follicular growth wave and the number of recruited follicles from this wave, accessory corpus luteum formation, the dominant follicle diameter and the spontaneous corpus luteum on the luteolitic administration day and subsequent synchronization rate and the interval between the $\mathrm{PGF}_{2} \alpha$ application and the estrus manifestation. Twenty crossbreed female cattle in diestrus, crossbred Holstein x Zebu and Simental x Zebu were allotted to four treatment groups, each containing five animals. In the treatment 1 the animals received $4 \mathrm{mg} \mathrm{IM}$. of buserelin at day 0 (day in which the treatment began) and $150 \mathrm{mg}$ of D (+) Cloprostenol at day 6 . In treatments 2, 3 and 4, the animals received 6, 8 and $10 \mathrm{mg}$ of buserelin, respectively, by the same application way, and 150 $\mathrm{mg}$ of $\mathrm{D}(+)$ Cloprostenol at day 6 . The ovarian activity was daily monitored, with the help of an ultra-sound machine, from day 0 to estrus or until day 11 on those animals, which did not answer to prostaglandin. The application of 8 or 10 mg of buserelin at an unknown phase of diestrus, in female crossbred cattle, promoted an emergence of a new follicular growth wave faster than the applications of 4 or $6 \mathrm{mg}$ of the same hormones, while the other evaluated parameters did not differ among treatments.
\end{abstract}

Key Words: follicular dynamic, buserelin, cloprostenol, estrus synchronization, cattle

\section{Introdução}

O hormônio liberador de gonadotrofinas $(\mathrm{GnRH})$ é um decapeptídeo, cujo principal alvo de ação é a hipófise anterior, onde se liga a sítios específicos (CLAYTON, 1989), liberando, principalmente, hormônio luteotrópico (LH) e, em menor intensidade, hormônio folículo estimulante (FSH) (BRITT et al., 1974; KALTENBACH et al., 1974).

A administração de buserelina, um agonista do
GnRH com, aproximadamente, 40 vezes sua potência (ORY et al., 1983), resulta em concentração sérica máxima de LH duas horas após sua aplicação, quantidade suficiente para estimular a ovulação dos folículos grandes (MACMILLAN et al., 1985; McDOUGALL et al., 1995).

Mensurações macroscópicas indicam que a administração de buserelina causa alteração da distribuição folicular nos ovários, diminuindo o número de folículos grandes pela indução da luteinização e, ou,

\footnotetext{
1 Doutorando Ciência Animal - UFMG.

2 Professor Titular, DZO - UFV.

3 Professor UNIFENAS.

4 Médico Veterinário.
} 
atresia (McNATTY et al., 1981; GUIBAULT et al., 1990). Contudo, observações histológicas indicam que o hormônio não influencia somente a distribuição entre classes de folículos, mas também o número total de folículos dos animais tratados (TWAGIRAMUNGO et al., 1994).

Administrações de buserelina sincronizam o desenvolvimento de nova onda folicular após a indução da ovulação ou luteinização de folículos maiores que 9 mm (STEVENSON et al., 1993; TWAGIRAMUNGO et al., 1994). Esse novo recrutamento folicular tem sido atribuído ao aumento das concentrações de FSH após a administração de buserelina, culminando na emergência de novo folículo dominante (ADAMS et al., 1992; BADINGA et al., 1992). Por sua vez, SILCOX et al. (1993) observaram que a habilidade do folículo dominante em ovular em resposta ao GnRH exógeno é dependente do estádio de seu desenvolvimento no instante do tratamento.

A formação de corpo lúteo acessório parece ser comum após a ovulação induzida pela aplicação de buserelina em vacas (MACMILLAN e THATCHER, 1991). A administração de buserelina prolonga a vida útil do corpo lúteo, protegendo-o parcialmente da luteólise espontânea e prolongando o ciclo estral (HENDERSON e McNATTY, 1975; MACMILLAN et al., 1985).A buserelina, indiretamente, aumenta o número de células luteais grandes e, conseqüentemente, o tamanho do corpo lúteo, resultando no aumento da concentração de progesterona (TWAGIRAMUNGO et al., 1994). Parece que o aumento do número de células luteais grandes nesses animais não é devido ao efeito direto sobre o corpo lúteo, visto que não se encontraram receptores específicos para GnRH nas células luteais de bovinos (BROW e REEVES, 1983; IRELAND et al., 1990). Entretanto, o fato de que injeções de LH ou hCG exógeno aumentam o número de células luteais grandes, associado a concomitante decréscimo no número de células luteais pequenas (NISWENDER et al., 1985; FARIN et al., 1988), indica que a ação do agonista de GnRH é mediada por meio de estimulação da secreção de gonadotrofinas endógenas, principalmente LH (CLARKE, 1989).

Estudos recentes têm sido feitos para controlar não somente a função do corpo lúteo, como também a qualidade do folículo ovulatório nos programas de sincronização de estro (WOLFENSON et al., 1994). A aplicação de buserelina, antes da indução da luteólise, aumenta a precisão da resposta ao estro (THATCHER et al., 1989; TWAGIRAMUNGO et al., 1992 a, b).
Objetivou-se com este experimento verificar o efeito de diferentes doses de buserelina sobre o dia de emergência da nova onda folicular, o número de folículos recrutados dessa onda, o diâmetro do folículo dominante no sexto dia após o tratamento hormonal, o diâmetro do folículo pré-ovulatório no dia do estro após aplicação de luteolítico, o diâmetro do corpo lúteo expontâneo no dia seis, a formação de corpo lúteo acessório, a taxa de sincronização do estro e o intervalo da aplicação de $\mathrm{PGF}_{2} \alpha$ à manifestação do estro.

\section{Material e Métodos}

O experimento foi realizado nas dependências da Fazenda Casa Nova, sediada no município de Araponga, Minas Gerais, no mês de fevereiro de 1996. Foram utilizadas 20 fêmeas bovinas mestiças Holandês x Zebu e Simental x Zebu (16 vacas secas e quatro novilhas) em diestro, com peso vivo acima de $300 \mathrm{~kg}$, escore corporal mínimo de 3,0 (FERGUSON e OTTO, 1989), ciclando regularmente, e sem anormalidades nos órgãos genitais, detectáveis ao exame ginecológico.

Constituíram-se quatro tratamentos contendo cinco animais cada (quatro vacas secas e uma novilha), sendo que, no tratamento 1 , os animais receberam $4 \mathrm{mg}$ de buserelina ${ }^{1}$, por via intramuscular (IM), no dia zero (dia de início do experimento), e $150 \mathrm{mg}$ IM de $\mathrm{D}(+)$ cloprostenol $^{2}$ no dia 6 . Nos tratamentos 2,3 e 4 , os animais receberam 6,8 e $10 \mathrm{mg}$ de buserelina no dia 0 e $150 \mathrm{mg}$ de $\mathrm{D}(+)$ cloprostenol no dia 6 , respectivamente. Todas as dosagens perfizeram volume total de $2 \mathrm{~mL}$. Quando necessário, era adicionada solução aquosa estéril em quantidade suficiente para completar esse volume.

A atividade ovariana foi monitorada diariamente, sempre no período da manhã, desde o dia zero até o dia do estro ou até o dia 11 pós-tratamento, naqueles animais que não responderam à prostaglandina. Os ovários foram monitorados, via retal, com auxílio de um transdutor linear de $5,0 \mathrm{MHz}$ e as imagens dos folículos e corpos lúteos foram observadas na tela do aparelho de ultra-som ALOKA SSD 500. Os folículos foram mapeados e seus diâmetros medidos após o congelamento da imagem mais representativa de cada estrutura. O diâmetro do corpo lúteo foi calculado pela maior distância entre suas bordas. Todos os exames foram gravados em vídeo-cassete e as imagens reexaminadas ao final do experimento, conferindo-se todas as medidas.

As observações visuais do estro foram feitas

1 Conceptal $^{\circledR}$ - Hoechst

2 Vegeglan ${ }^{\circledR}$ - Serono 
1228 Rev. bras. zootec.

diariamente até o sexto dia, das 6 às 8 h e 17 às 19 h, e a partir do dia seis, ininterruptamente, de 5 às $22 \mathrm{~h}$, e também com o uso de um rufião e uma fêmea androgenizada portando buçal marcador. Registrou-se a hora em que o estro começou, ou seja, quando os animais apresentaram reflexo de imobilização à monta.

As amostras de sangue foram coletadas em todos os animais nos dias zero, seis e no dia do estro, utilizando-se tubos vacuolizados para punção da veia ou artéria coccígeas, ficando em temperatura ambiente por 60 a 120 minutos, quando foram centrifugadas a $3.000 \mathrm{rpm}$, durante 15 minutos. O soro foi armazenado a $-18^{\circ} \mathrm{C}$.

As concentrações séricas de progesterona foram dosadas por radioimunoensaio em fase sólida com o uso de kit comercial ${ }^{3}$.

O experimento foi realizado no delineamento inteiramente casualizado. As variáveis número de folículos recrutados da nova onda folicular, número de animais com corpo lúteo acessório, diâmetro do folículo dominante e do corpo lúteo espontâneo no dia seis, diâmetro do folículo pré-ovulatório no dia do estro, intervalo entre a aplicação da $\mathrm{PGF}_{2} \alpha$ e o estro e o dia da emergência da nova onda folicular foram estudadas pela análise de regressão linear, admitindo-se nível de $5 \%$ de probabilidade.

\section{Resultados e Discussão}

Todos os animais estavam ciclando e na fase de diestro, pois apresentavam concentração sérica de progesterona no dia zero (dia em que o tratamento foi iniciado), $\geq 1,0 \mathrm{ng} / \mathrm{mL}$ (Tabela 1), características que, segundo NAWITO et al. (1977) e MACMILLAN et al., (1985), os tornavam aptos a responder ao tratamento hormonal. A média do peso vivo e a amplitude do escore corporal foram $425,4 \mathrm{~kg}$ e 3,0 a 4,0 , respectivamente.

As diferentes doses de buserelina foram eficientes em inibir ou coincidiram com a não-manifestação do estro espontâneo entre os dias zero e seis, conforme reportado anteriormente por TWAGIRAMUNGO et al. (1992 a, b). Essa inibição se deve à alteração no desenvolvimento folicular, induzindo luteinização e, ou, atresia após sua aplicação (GUIBAULT et al., 1990; MACMILLAN et al., 1985).

Um animal que recebeu $8 \mathrm{mg}$ de buserelina e dois que receberam $10 \mathrm{mg}$ tinham ovulado no dia um (Tabela 2), de modo semelhante ao observado por McDOUGALL et al., (1995), os quais reportaram que a administração de um análogo de GnRH poderia
Tabela 1 - Concentrações séricas de progesterona nos dias 0 (dia em que iniciou o tratamento), 6 e no dia do estro dos animais que receberam diferentes doses de buserelina

Table 1- Progesterone seric concentration at $d 0$ (the beginning of the experiment) and 6 and estrus day of animals receiving diferents buserelin doses

\begin{tabular}{lccc}
\hline $\begin{array}{l}\text { Doses de } \\
\text { buserelina }(\mathrm{mg}) \\
\text { Buserelin doses }\end{array}$ & \multicolumn{3}{c}{$\begin{array}{c}\text { Concentração de } \\
\text { progesterona }(\mathrm{ng} / \mathrm{mL}) \\
\text { Progesterone } \\
\text { concentration }(n g / m L)\end{array}$} \\
\cline { 2 - 4 } & Dia0 & Dia 6 & Dia do estro \\
& Day 0 & Day 6 & Estrus day \\
\hline 4 & $3,42(2,1-5,3)$ & $5,36(4,7-5,7)$ & $0,36(0,1-0,7)$ \\
6 & $3,82(2,5-5,1)$ & $3,95(1,4-6,8)$ & $0,30(0,1-0,5)$ \\
8 & $2,96(1,4-6,9)$ & $3,32(1,1-5,7)$ & $0,16(0,0-0,4)$ \\
10 & $3,20(1,4-4,5)$ & $3,94(1,6-6,8)$ & $0,26(0,1-0,5)$ \\
\hline
\end{tabular}

liberar quantidade suficiente de $\mathrm{LH}$, estimulando a ovulação dos folículos grandes.

Os animais que receberam 4 ou 6 mg não apresentaram ovulação induzida pela buserelina. Estes resultados não devem ser atribuídos ao efeito das diferentes doses, em virtude do desconhecimento do dia do diestro em que os animais se encontravam, pois um único exame ultra-sonográfico no dia zero não é suficiente para se determinar o estádio de desenvolvimento folicular (BO et al., 1995). As fêmeas que ovularam em resposta à buserelina apresentaram folículos maiores que $1,4 \mathrm{~cm}$ de diâmetro no dia zero, pois provavelmente tratava-se do folículo dominante daquela onda. Houve formação de corpo lúteo acessório nesses três animais, sendo identificado no dia três. Essa estrutura luteal secundária regrediu após a aplicação de luteolítico no dia seis, concordando com os estudos de PURSLEY et al. (1995), sugerindo que, nesse dia, a mesma já possuía receptores suficientes para $\mathrm{PGF}_{2} \alpha$.

A administração de buserelina, durante o diestro, parece ter induzido o surgimento de nova onda de crescimento folicular até cinco dias após sua aplicação (Tabela 2). Uma das possíveis formas de sua ação pode ter sido feita por meio da eliminação do efeito supressivo do folículo dominante sobre a emergência de nova onda, naqueles animais que o possuíam no dia do tratamento (MACMILLAN e THATCHER, 1991), ou induzindo-se a luteinização e, ou, atresia dos folículos menores (TWAGIRAMUNGO et al., 1994).

Os dias de emergência da nova onda de crescimento folicular, após a aplicação de 8 e $10 \mathrm{mg}$ de buserelina (Figura 1), são compatíveis com os achados de outros autores (PURSLEY et al., 1995). Com base no fato de que a emergência de nova onda é precedida um ou dois dias, por um aumento da con-

${ }^{3}$ COAT-A-COUNT ${ }^{\circledR}-$ DPC 
COSTA et al.

Tabela 2 - Dinâmica folicular, diâmetro do corpo lúteo expontâneo (cm), formação de corpo lúteo acessório, taxa de sincronização (\%) e intervalo da aplicação de $\mathrm{PGF}_{2} \alpha$ ao estro (horas) de animais tratados com diferentes doses de buserelina

Table 2 - Follicular dynamic, spontaneous corpus luteum diameter (cm), accessory corpus luteum formation, synchronization rate (\%) and time interval from $\mathrm{PGF}_{2} \alpha$ to onset of estrus (hours) of animals treated with different buserelin doses

\begin{tabular}{|c|c|c|c|c|}
\hline \multirow[t]{2}{*}{$\begin{array}{l}\text { Parâmetro } \\
\text { Parameter }\end{array}$} & \multicolumn{4}{|c|}{$\begin{array}{c}\text { Doses de buserelina }(\mathrm{mg}) \\
\text { Buserelin doses }\end{array}$} \\
\hline & 4 & 6 & 8 & 10 \\
\hline Número de animais & 5 & 5 & 5 & 5 \\
\hline Number of animals & & & & \\
\hline $\begin{array}{l}\text { № de animais que tiveram emergência de nova } \\
\text { onda folicular do dia } 1 \text { a } 6\end{array}$ & 5 & 5 & 5 & 5 \\
\hline $\begin{array}{l}\text { Animals with new follicular wave emergence (days } 1 \text { to } 6) \\
\text { № de folículos recrutados da nova onda (> } 4 \mathrm{~mm})\end{array}$ & $3,2+1,09$ & $4,0+1,58$ & $3,6+0,89$ & $3,0+1,22$ \\
\hline $\begin{array}{l}\text { Number of recruited follicles of new wave }(>4 \mathrm{~mm}) \\
\text { Diâmetro do folículo dominante no dia } 6(\mathrm{~cm}) \\
\text { Dominant follicle diameter at d } 6(\mathrm{~cm})\end{array}$ & $0.88 \pm 0.22$ & $1,01 \pm 0,27$ & $0,92 \pm 0,29$ & $0,74 \pm 0,16$ \\
\hline $\begin{array}{l}\text { Diâmetro do folículo pré-ovulatório no dia do estro }(\mathrm{cm}) \\
\text { Pre ovulatory follicle diameter at estrus }(\mathrm{cm})\end{array}$ & $1,47 \pm 0,16$ & $1,52 \pm 0,24$ & $1,54+0,36$ & $1,39 \pm 0,35$ \\
\hline $\begin{array}{l}\text { Diâmetro do CL no dia } 6(\mathrm{~cm}) \\
\text { Spontaneous CL diameter at } d 6(\mathrm{~cm})\end{array}$ & $2,46+0,74$ & $2,6+0,99$ & $2,6+0,48$ & $2,54+1,17$ \\
\hline $\begin{array}{l}\text { № de animais com CL acessório no dia } 6 \\
\text { Number of animals with accessory CL at d } 6\end{array}$ & 0 & 0 & 1 & 2 \\
\hline $\begin{array}{l}\text { Taxa de sincronização do estro (\%) } \\
\text { Estrus sinchronization rate (\%) }\end{array}$ & 60 & 60 & 100 & 100 \\
\hline $\begin{array}{l}\text { Intervalo da aplicação de } \mathrm{PGF}_{2} \alpha \text { e o estro (horas) } \\
\text { Time interval from } P G F_{2} \alpha \text { onset to estrus (hours) }\end{array}$ & $75,19 \pm 17,28$ & $55,9 \pm 29,83$ & $72,42 \pm 39,07$ & $81,26 \pm 34,76$ \\
\hline
\end{tabular}

centração de FSH (ADAMS et al., 1992), e a adminstração de buserelina induz a liberação de LH e FSH (CHENAULT et al., 1990), acredita-se que a emergência observada nestes tratamentos foi induzida pela aplicação hormonal ou por causa da liberação de FSH endógeno, que ocorre, normalmente, depois que o folículo dominante sofre ovulação ou atresia (KO et al., 1991; ADAMS et al., 1992).

Os efeitos observados após a aplicação de 4 ou 6 mg de buserelina parecem ser decorrentes da efêmera elevação de LH e FSH, causada após a administração dessas baixas doses (NAWITO et al., 1977; CHENAULT et al., 1990).

A seleção do folículo pré-ovulatório, nos animais que receberam 8 ou $10 \mathrm{mg}$ de buserelina, já tinha ocorrido no dia da aplicação do luteolítico, conforme reportado por TWAGIRAMUNGO et al. (1994), sendo que o folículo pré-ovulatório, no dia do estro, foi o folículo dominante da nova onda induzida pelo tratamento no dia zero, corroborando com SAVIO et al. (1990) e FORTUNE (1993), os quais reportaram que o folículo dominante, quando ainda está crescendo, é capaz de ovular em resposta à indução da luteólise com cloprostenol. No entanto, não se observou diferença na precisão da manifestação do estro entre os tratamentos (Figura 2).

O número de folículos recrutados da nova onda (Tabela 2) foi semelhante em todos os tratamentos,

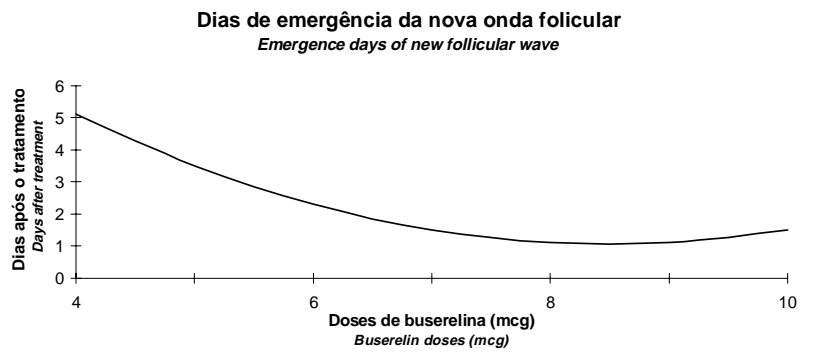

Figura 1 - Dias de emergência da nova onda folicular após o tratamento com diferentes doses de buserelina. $\hat{\mathrm{Y}}=15,50-3,4 D^{\star \star}+2,20 D^{2^{* \star}}(P<0,01) R^{2}=$ 0,9799 .

Figure 1 - Emergence days of new follicular wave after treatment with different buserelin doses. $\hat{Y}=15,50-3,4 D^{* *}+$ $2,20 D^{2^{* \star}}(P<0,01) R^{2}=0,9799$.

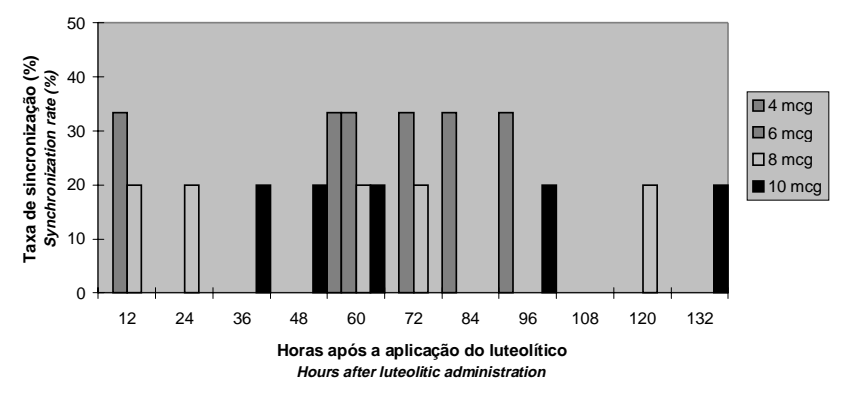

Figura 2 - Distribuição do estro, a cada 12 horas, após a aplicação de $\mathrm{PGF}_{2} \alpha$ no dia 6 , precedida de diferentes doses de buserelina no dia 0 (dia em que iniciou o tratamento).

Figure 2 - Estrus distribution, in 12-12 hours, after $P G F_{2} \alpha$ administration at $d 6$, proceed of diffferent buserelin doses at $d 0$ (the beginning of the experiment). 
estando dentro da média encontrada por outros autores (FORTUNE et al., 1988; SIROIS e FORTUNE, 1988).

Os diâmetros do folículo dominante no dia seis, do folículo pré-ovulatório no dia do estro e do corpo lúteo no dia seis (Tabela 2) não foram diferentes entre os tratamentos $(\mathrm{P}>0,05)$. Estas diferenças, caso existam, podem ser evidenciadas se houver maior número de animais em cada grupo. Da mesma forma, a taxa de sincronização e o intervalo entre a aplicação de $\mathrm{PGF}_{2} \alpha$ e a manifestação do estro não diferiram entre os tratamentos $(\mathrm{P}>0,05)$.

\section{Conclusões}

A aplicação de 8 ou $10 \mathrm{mg}$ de buserelina em uma fase indeterminada do diestro, em fêmeas bovinas mestiças, promove emergência de nova onda de crescimento folicular mais rápida que aplicações de 4 ou $6 \mathrm{mg}$ do mesmo hormônio.

\section{Referências Bibliográficas}

ADAMS, G.P., MATTERI, R.L., GINTHER, O.J. 1992. Effect of progesterone on growth of ovarian follicles, emergence of follicular waves and circulating FSH in heifers. J. Reprod. Fertil. 95:627-640.

BADINGA, L., DRIANCOURT, M.A., SAVIO, J.D. et al. 1992. Endocrine and ovarian responses associated with the first wave dominant follicle in cattle. Biol. Reprod., 47:871.

BO, G.A., ADAMS, G.P., PIERSON, R.A. et al. 1995. Exogenous control of follicular wave emergence in cattle. Theriogenology, 43:31-40.

BRITT, J.H., KITTOK, R.J., HARRISON, D.S. 1974. Ovulation, estrus and endocrine response after GnRH in early postpartum cows. J. Anim. Sci., 39:915-919.

BROWN, J.L., REEVES, J.J. 1983. Absence of specific luteinizing hormone releasing hormone receptors in ovine, bovine and porcine ovaries. Biol. Reprod., 29:1179-1184.

CHENAULT, J.R., KRATZER, D.D., RZEPKOWSKI, R.A., et al. 1990. LH and FSH response of holstein heifers to ferterelin acetate, gonadorelin and buserelin. Theriogenology, 3:81-98.

CLARKE, I.J. 1989. The GnRH/gonadotropin axis in the ewe, cow and sow. Domest. Anim. Endocrinol., 6:1.

CLAYTON, R.N. 1989. Gonadotrophin-releasing hormone: its actions and receptors. J. Endocrinol., 120:11-19.

FARIN, C.E., MOELLER, C.L., MAYAN, H. et al. 1988. Effect of luteinizing hormone and human chorionic gonadotropin on cell populations in the ovine corpus luteum. Biol. Reprod., 38:413-421.

FERGUSON, J.D., OTTO, K.A. 1989. Managing body condiction in dairy cows. Proc. Cornell Nutr. Conf. Feed Manuf., p.75-87.

FORTUNE, J.E., SIROIS, J., QUIRK, S.M. 1988. The growth and differentiation of ovarian follicles during the bovine estrous cycle. Theriogenology, 29:95-110.

FORTUNE, J.E. 1993. Follicular dynamics during the bovine estrous cycle: A limiting factor in improvement of fertility? Anim. Reprod. Sci., 33:111-125.

GUIBAULT, L.A., LUSIER, J.G., GRASSO, F. et al. 1990. Influence of a GnRH analogue on follicular dynamics in cows pretreated or not with FSH-P. Theriogenology, 33:240.

HENDERSON, K.M., McNATTY, K.P. 1975. A biochemical hypothesis to explain the mechanism of luteal regression. Prostaglandins, 9:779.
IRELAND, J.J., MILVAE, R.A., MARTIN, T.L. et al. 1990. Effect of histone $\mathrm{H} 2 \mathrm{a}$ on progesterone production by bovine luteal cells. Biol. Reprod., 43:1058.

KALTENBACH, C.C., DUNN, T.G., KISER, T.E. et al. 1974. Release of FSH and LH in beef heifers by synthetic gonadotrophin releasing hormone. J. Anim. Sci., 38:357-362.

KO, J.C.H., KASTELIC, J.P., Del CAMPO, M.R. et al. 1991. Effect of a dominant follicle on ovarian follicular dynamics during the cycle in heifers. J. Reprod. Fert., 91:511-519.

MACMILLAN, K.L., DAY, A.M., TAUFA, V.K. et al. 1985. Effect of an agonist of gonadotrophin releasing hormone in cattle. I hormone concentrations and oestrous cycle length. Anim. Reprod. Sci., 8:203-212.

MACMILLAN, K.L., THATCHER, W.W. 1991. Effect of an agonist of gonadotropin-releasing hormone on ovarian follicles in catlle Biol. Reprod., 45:883-889.

McDOUGALL, S., WILLIAMSON, N.B., MACMILLAN, K.L. 1995. GnRH induces ovulation of a dominant follicle in primiparous dairy cows undergoing anovulatory follicle turnover Anim. Reprod. Sci., 39:205-214.

McNATTY, K.P., GIBB, M., DOBSON, C., et al. 1981. Evidence that changes in luteinizing hormone secretion regulate the growth of the preovulatory follicle in the ewe. J. Endocrin., 90:375.

NAWITO, M., SCHALLENBERGER, E., SCHAMS, D. 1977. Releasing of lutropin (LH) and follitropin (FSH) in cattle after administration of a new gonadoliberin $(\mathrm{GnRH})$ analogue in comparison with the gonadoliberin dacapeptide. Theriogenology, 7:277-287.

NISWENDER, G.D., SCHWALL, R.H., FITZ, C.E. et al. 1985. Regulation of luteal function indomestic ruminants: new concepts. Rec. Prog. Horm. Res., 41:101-151.

ORY, S.J. 1983. Clinical uses of luteinizing hormone-releasing hormone. Fertil. Steril., 39:577-590

PURSLEY, J.R, MEE, M.O., WILTBANK, M.C. 1995. Synchronization of ovulation in dairy cows using $\mathrm{PGF}_{2}$ a and GnRH. Theriogenology, 44:915-923.

SAVIO, J.P., BOLAND,M.P., HYNES, N. et al. 1990. Will the first dominant follicle of the estrous cycle of heifers ovulate following luteolysis on day 7 ? Theriogenology, 33:677-687.

SILCOX, R.W., POWELL, K.L., KISER, T.E. 1993 Abiblity of dominant follicle (DF) to respond to exogenous $\mathrm{GnRH}$ administration on their stage of development. J.Anim. Sci., 71:219.

SIROIS, J., FORTUNE, J.E. 1988. Follicular dynamics during the estrous in heifers monitored by real-time ultrasonography. Biol. Reprod., 39:308-317.

STEVENSON, J.S., PHATAK, A.P., RETTMER, I. et al. 1993. Postinsemination administration of receptal: follicular dynamics, duration of cycle, hormonal responses, and pregnancy rates. J. Dairy Sci., 76:2536-2547.

THATCHER, W.W., MACMILLAN, K.L., HANSEL, P.J. et al. 1989. Concepts for regulation of corpus luteum function by the conceptus and ovarian follicles to improve fertility. Theriogenology, 31:149-163.

TWAGIRAMUNGO, H., GUILBAULT, L.A., PROULX, J. et al. 1992a. Synchronization of estrus and fertility in beef cattle with two injections of buserelin and prostaglandin. Theriogenology, 38:1131-1144.

TWAGIRAMUNGO, H., GUILBAULT, L.A., PROULX, J. et al. 1992b. Influence of an agonist of gonadotropin-releasing hormone (Buserelin) on estrus synchronization and fertility in beef cows. J. Anim. Sci., 70:1904-1910.

TWAGIRAMUNGO, H., GUILBAULT, L.A., PROULX, J. et al. 1994. Histological populations and atresia of ovarian follicles in postpartum cattle treated with na agonist of gonadotropin-releasing hormone J. Anim. Sci., 72:192-200.

WOLFENSON, D., THATCHER, W.W., SAVIO, J.D. et al. 1994. The effect of a GnRH analogue on the dynamics of follicular development and synchronization of estrus in lacting cyclic dairy cows. Theriogenology, 42:633-644.

Recebido em: 14/05/98 Aceito em: 02/08/99 